\title{
Percepção dos Clientes sobre a Qualidade do Serviço de Provedores Formalmente Avaliados nos Modelos CMM®/CMMI®
}

\author{
Rodrigo Pinheiro dos Santos, Kathia Marçal de Oliveira, Wander Pereira da Silva
}

Programa de Pós-Graduação em Gestão do Conhecimento e da Tecnologia da Informação - Universidade Católica de Brasília (UCB)

SGAN 916 Norte AV. W5 - 70.790-160 - Brasília - DF - Brasil

rodpin@terra.com.br, \{kathia, wander\}@ucb.br

\begin{abstract}
Recently, several companies decided to adopt models like CMMI® and MPS.BR, to assure the quality in their software process. However, how much are their customers satisfied? Which are their perceptions about the service quality offered by these providers? That is why this work proposes a provided service quality analysis by companies formally evaluated on $C M M ® / C M M I ®$. In order to deal with this demand, it was built an instrument based on a largely used quality of service evaluation model (the SERVQUAL). The results show a considerable dissonance between the expectations of customers and their perceptions about the provided services.
\end{abstract}

Resumo. Recentemente muitas empresas decidiram pela adoção de modelos como o CMMI® e MPS.BR para garantir qualidade de seus processos de software. No entanto, quão satisfeitos estão os clientes dessas empresas? Qual a percepção sobre a qualidade do serviço realizado por esses provedores? $O$ presente trabalho investiga a percepção da qualidade dos serviços providos, por empresas formalmente avaliadas nos modelos CMM®/CMMI®. Para isso foi utilizado um instrumento baseado em um modelo de avaliação da qualidade de serviço largamente empregado (o SERVQUAL). Os resultados mostram um desalinhamento negativo entre as expectativas dos clientes e a sua percepção em relação aos serviços prestados.

\section{Introdução}

Em busca de uma maior agilidade, redução de custos e em muitos casos melhores serviços, várias empresas optaram por terceirizar parte ou até completamente os seus serviços de TI (Tecnologia da Informação). Neste cenário, um dos processos de TI que mais foram atingidos é o desenvolvimento de software. Com contratos de prestação de serviços in-house ou com as chamadas fábricas de software, as empresas abriram mão de parte ou de todo o controle do processo de desenvolvimento e passaram a se concentrar nos produtos de software desejados. A partir desta dedicação, logo a cobrança por melhores resultados na qualidade do serviço fornecido aumentou.

Em resposta a esta situação, a indústria de desenvolvimento de software agenciou o surgimento de padrões e modelos de melhorias relacionados ao processo de desenvolvimento, tais como: CMM® (Capability Maturity Model) [Paulk et al 1993], 
CMMI® (CMM Integration) [Carnegie Mellon University 2002a, 2002b] e MPS.BR [SOFTEX 2006]. Segundo o SEI (Software Engineering Institute) [CMMI 2006], a adoção do $\mathrm{CMM}{ }^{\circledR} / \mathrm{CMMI}{ }^{\circledR}$ pelas empresas traz bons resultados, principalmente, relacionados ao prazo de entrega, redução de defeitos e custos.

Porém, existe certa divergência nos estudos a respeito de um quesito importante: a satisfação do cliente. Os defensores dos modelos e padrões citados afirmam que a melhoria nos processos acarreta em ampliação da qualidade do produto e, por conseguinte na satisfação do usuário/cliente [Ahren; Clouse; Turner 2003; Associação Brasileira de Normas Técnicas 2003]. Outra linha defende que a satisfação do usuário/cliente pode envolver diversos outros atributos como manutenibilidade, portabilidade, disponibilidade, entre outros, que não são endereçados diretamente por tais modelos [Bevan 1999].

Segundo Cronin e Taylor (1992), a satisfação do cliente é apenas um dos componentes de um conceito maior: a qualidade de serviço. Alguns autores propuseram instrumentos para a avaliação dessa qualidade, sendo que os trabalhos de maior destaque foram realizados por Parasuraman, Zeithaml e Berry (1985; 1994), que propuseram um instrumento denominado SERVQUAL. Esse instrumento avalia a qualidade do serviço como sendo a diferença entre as expectativas dos clientes e a percepção que estes obtiveram após a prestação do serviço [Parasuraman; Zeithaml; Berry 1985]

Este trabalho tem por objetivo realizar uma avaliação da qualidade dos serviços providos por empresas formalmente avaliadas nos modelos $\mathrm{CMM} / \mathrm{CMMI}$, utilizando um instrumento que foi concebido com base no SERVQUAL e uma de suas variantes para a área de sistema de informação o IS-SERVQUAL [Kettinger; Lee 1997].

Nas seções seguintes são apresentados conceitos básicos sobre os modelos de maturidade CMM®/CMMI® (seção 2) e avaliação da qualidade de serviço (seção 3), utilizados para a elaboração do instrumento de avaliação (seção 4). Finalmente, são apresentados parte dos resultados alcançados (seção 5) e as conclusões deste trabalho (seção 6).

\section{CMM®/CMMI®}

Segundo Paulk et al (1993), o modelo de capacidade de maturidade para software (SWCMM) "foi desenvolvido para guiar as organizações de software a selecionar estratégias de melhoria de processos, determinando a atual maturidade dos processos $\mathrm{e}$ identificando os poucos itens mais críticos para a qualidade de software e melhoria de processos" [Paulk et al, 1993, p.5].

Com o grande sucesso do modelo SW-CMM®, e o surgimento de novos modelos de melhoria como os de aquisição de software (SA-CMM), gerenciamento de força de trabalho (P-CMM) e desenvolvimento de produto e processo de software integrados (IPD-CMM), surgiu a necessidade de se agrupar alguns destes modelos para facilitar a aplicação nas organizações que utilizam mais de um [Carnegie Mellon University 2002a]. Assim surgiu o CMMI® (Capability Maturity Model Integration) como um conjunto de boas práticas que pretende prover uma visão integrada da maturidade de processos de desenvolvimento [Chrissis 2003]. 
Este modelo é organizado em duas representações: a estágio e a contínua. Basicamente a diferença entre as duas representações é o foco dado aos níveis. Na representação por estágio os níveis de maturidade guiam as áreas de processo, já na contínua os níveis são chamados de capacidade e o foco é nas práticas específicas e genéricas [KULPA; JOHNSON, 2003]. Na representação em estágio, o modelo está estruturado em cinco níveis de maturidade. Cada nível de maturidade, com exceção do inicial, contém áreas de processos que são dirigidas por objetivos genéricos e específicos, e estes são alcançados por práticas genéricas e específicas. Ao todo o CMMI SE/SW 1.1 possui 22 áreas de processo, 157 metas e 539 práticas [Carnegie Mellon University 2002a].

Em um levantamento realizado em fevereiro de 2007, que sintetizou informações diretamente do site do SEI, do site de uma das avaliadoras oficiais no Brasil (ISD Brasil) e de um estudo do Ministério da Ciência e Tecnologia (MCT) [Brasil 2006], foi verificado que no cenário nacional existem 83 centros avaliados no CMM®/CMMI®. A palavra centro foi utilizada, pois algumas organizações possuem mais de um departamento avaliados em um dos dois modelos.

\section{Qualidade de Serviço}

A partir das últimas décadas, motivadas pela crescente competição do mercado global, as empresas iniciaram uma busca por qualidade, evoluindo seus equipamentos, processos e pessoas que atuam na produção de um produto ou prestação de um serviço. Como destacado por Slack et al (1996, p. 549) a "boa qualidade deriva em redução de custos, maior escala e o mais importante clientes satisfeitos".

Porém, mesmo considerando a satisfação destes clientes um dos fatores mais importantes para a manutenção do negócio, atingir esta meta não é nada trivial. No caso dos serviços a situação complica-se um pouco mais, pois estes possuem características que por si só são agravantes de medição e adequação da qualidade [Parasuraman; Zeithaml; Berry 1985]. Grönroos (1993) identificou em seu trabalho que a maioria dos serviços possuía quatro propriedades básicas, que são: os serviços são mais ou menos intangíveis; os serviços são atividades ou uma série de atividades em vez de coisas; os serviços são, pelo menos até certo ponto, produzidos e consumidos simultaneamente; e, o cliente participa do processo de produção, pelo menos até certo ponto.

Uma das grandes dificuldades das empresas em direcionar a qualidade para serviços é que em muitos casos, estas estão preocupadas com seus fatores internos, e pouco dão atenção à percepção do cliente sobre os serviços [Grönroos 1993]. Por este motivo, as medições de qualidade deveriam ser realizadas com foco no cliente. Franceschini (2001) relata que a qualidade de serviços com foco no cliente apresenta três critérios de análise:

- Qualidade Oferecida - é o resultado da concepção do serviço;

- Qualidade Esperada - é a que satisfaz a expectativa do usuário;

- Qualidade Percebida - é aquela observada pelo cliente.

A qualidade percebida do serviço pode ser definida como sendo a forma em que os clientes e/ou usuários "percebem" um produto ou serviço. Onde o termo "perceber", neste contexto, está relacionado à observação que cada indivíduo realizou no 
recebimento da prestação do serviço [Rados, 2005], [Slack et al 1996], [Grönroos 1993].

Segundo Slack et al (1996), o grau de medição da qualidade de serviço é determinado através da diferença entre qualidade percebida $(\mathrm{P})$ e a qualidade esperada (E), ou seja, as lacunas entre estas visões é que irão gerar uma boa ou pobre percepção do cliente quanto ao serviço. Assim, quanto maior for a diferença positiva entre a qualidade percebida e a esperada (P-E), melhor é a percepção sobre a qualidade de serviço.

\subsection{O Modelo SERVQUAL}

Para a avaliação da qualidade de serviço, são destacados os trabalhos apresentados por Parasuraman, Zeithaml e Berry (1985, 1988, 1991a, 1991b, 1994). Esses autores desenvolveram um instrumento, denominado SERVQUAL, que foi dividido em duas partes um para análise da qualidade esperada e outra para o julgamento da qualidade percebida. Para avaliação da qualidade esperada e da qualidade percebida foi elaborado um conjunto de questões distribuídas em 5 dimensões de qualidade (Tabela 1). Cada questão é escrita tanto para qualidade esperada como para percebida totalizando 44 questões (22 para qualidade esperada e 22 para percebida) que deveriam ser respondidas em uma escala ordinal de 04 itens [Parasuraman; Zeithaml; Berry 1988].

Tabela 1. Dimensões do Modelo SERVQUAL

\begin{tabular}{|l|l|}
\hline \multicolumn{1}{|c|}{ Dimensão } & \multicolumn{1}{c|}{ Descrição } \\
\hline Tangíveis & Facilidades físicas, equipamentos e aparência do pessoal. \\
\hline Confiabilidade & Habilidade de executar o serviço prometido de forma segura e correta. \\
\hline $\begin{array}{l}\text { Capacidade de } \\
\text { Resposta }\end{array}$ & Voluntariedade em auxiliar o cliente e prover prontidão no serviço \\
\hline Segurança & $\begin{array}{l}\text { Conhecimento e cortesia dos empregados e sua habilidade de inspirar } \\
\text { confiança e certeza. }\end{array}$ \\
\hline Empatia & $\begin{array}{l}\text { Dispor de atenção individualizada da empresa para com os seus } \\
\text { clientes }\end{array}$ \\
\hline
\end{tabular}

Este modelo foi amplamente discutido [Van Dyke; Kappelman; Prybutok 1997; Pitt; Watson; Prybutok 1997; Kettinger; Lee 1997] e utilizado em diferentes contextos: em avaliações de serviços bancários [Paiva 2001], de complexo poliesportivo [Reis 2001], para a manutenção de software de sistemas de informações [Kang; Bradley 2002], serviços de teste de software com processos baseados no CMMI® [Quintela et. al 2006], entre outros. Por se tratar de um modelo genérico, em cada uma das aplicações foi realizada uma adaptação das perguntas de forma a refletir o novo domínio avaliado.

Em seu estudo Kettinger e Lee (1997), propõem uma derivação do SERVQUAL mais adaptado a área de SI (Sistemas de Informação). Neste novo modelo foi retirada a dimensão de tangíveis, que segundo os autores, possuiria baixa confiabilidade, e foi reduzido o número de itens para 26 [Kettinger; Lee 1997].

\section{Elaboração do Instrumento de Avaliação}

Para avaliação da qualidade de serviço dos provedores CMM ${ }^{2} / \mathrm{CMMI} ®$ foram consideradas três premissas principais:(i) utilizar o instrumento SERVQUAL e ISSERVQUAL como referência, pelo fato do SERVQUAL ser um modelo extensamente utilizado na área de qualidade de serviço, e o IS-SERVQUAL ser uma customização do 
modelo genérico para a área de sistemas de informação, que é mais próximo da área de desenvolvimento software; (ii) utilizar o CMMI® SE/SW 1.1 como referência, considerando que o $\mathrm{CMM}{ }^{\circledR}$ foi descontinuado e que as empresas avaliadas neste modelo devem migrar para o atual; e, (iii) buscar um número limitado de questões de forma a simplificar a avaliação.

Baseado na primeira premissa foi realizado um mapeamento das questões do modelo SERVQUAL e IS-SERVQUAL. Foi possível observar, que além das questões da dimensão de tangíveis que realmente não faziam parte do IS-SERVQUAL, em todas as outras dimensões pelo menos uma questão do SERVQUAL não estava presente no IS-SERVQUAL. Esse mapeamento foi feito, pois considerando que o modelo ISSERVQUAL já é uma customização para a área de sistemas de informação, no momento da elaboração das questões deveria se optar pela customização presente neste instrumento. Foram escolhidas também às mesmas dimensões do modelo ISSERVQUAL, isto é, excluindo-se a dimensão de tangíveis, uma vez que as empresas estão optando pelo modelo de terceirização off-shore de desenvolvimento, ou seja, do tipo fábrica de software, que em muitos dos casos os clientes não tem contato com as instalações do provedor.

Para atender a segunda premissa foi necessário analisar todas as áreas de processo, objetivos específicos e práticas específicas do CMMI, buscando identificar o que poderia ser percebido pelo cliente nas dimensões de qualidade definidas pelo modelo SERVQUAL. Esta análise será apresentada na seção 4.1. Baseado nas duas primeiras premissas foi elaborado o primeiro instrumento de avaliação de forma a adaptar as questões do modelo original aos conceitos do CMMI®. Finalmente, esse questionário foi refinado de forma a atender um número limitado de questões que respeitasse a última premissa. A seção 4.2 apresenta a construção final do questionário.

\subsection{Análise do CMMI em relação ao SERVQUAL e IS-SERVQUAL}

Para realizar a análise do CMMI® em relação aos modelos de avaliação de qualidade de serviço SERVQUAL e IS-SERVQUAL, foi considerado os objetivos e práticas dos níveis 2 e 3 , considerando que esses níveis são a base principal para a gerência e atividades de engenharia de software.

O primeiro passo foi analisar as áreas de processo do $\mathrm{CMMI}{ }^{\circledR}$ com às dimensões de qualidade de serviço considerando as descrições das mesmas, verificando assim quais dimensões tinham uma melhor correlação com cada área. A tabela 2 apresenta esse mapeamento considerando as áreas do nível 2, apresentando a justificativa do por quê de cada área está relacionada com uma determinada dimensão. Posteriormente, foram relacionadas não somente as áreas e sim as práticas específicas de cada área com as dimensões de qualidade de serviço, como exemplificado na Tabela 3, no qual o cruzamento mostra a justificativa do mapeamento, ou seja, o porquê da prática está relacionada com a dimensão. 
Tabela 2. Exemplo de mapeamento CMMI® nível 2 e Dimensões.

\begin{tabular}{|c|c|c|c|c|}
\hline \multirow[b]{2}{*}{ CMMI® } & \multicolumn{4}{|c|}{ Dimensões de Qualidade de Serviço } \\
\hline & Confiabilidade & $\begin{array}{c}\text { Capacidade de } \\
\text { Resposta }\end{array}$ & Segurança & Empatia \\
\hline $\begin{array}{l}\text { Gerência de } \\
\text { Requisitos } \\
\text { (REQM) }\end{array}$ & $\begin{array}{l}\text { Gerenciar os } \\
\text { requisitos }\end{array}$ & & $\begin{array}{c}\text { Identificar } \\
\text { inconsistência } \\
\text { entre os } \\
\text { requisitos e } \\
\text { produtos de } \\
\text { trabalho }\end{array}$ & $\begin{array}{c}\text { Obter } \\
\text { entendimento } \\
\text { dos requisitos }\end{array}$ \\
\hline $\begin{array}{l}\text { Planejamento de } \\
\text { Projeto (PP) }\end{array}$ & $\begin{array}{c}\text { Estabelecer e manter } \\
\text { planos das atividades } \\
\text { do projeto }\end{array}$ & & & \\
\hline $\begin{array}{l}\text { Planejamento e } \\
\text { Monitoração } \\
\text { (PMC) }\end{array}$ & $\begin{array}{c}\text { Viabilizar } \\
\text { acompanhamento do } \\
\text { progresso do projeto }\end{array}$ & $\begin{array}{c}\text { Viabilizar } \\
\text { acompanhamento } \\
\text { do progresso do } \\
\text { projeto }\end{array}$ & & \\
\hline $\begin{array}{l}\text { Gerenciamento de } \\
\text { Acordos com } \\
\text { Fornecedores } \\
(\mathrm{SAM})\end{array}$ & $\begin{array}{l}\text { Gerenciar a aquisição } \\
\text { de produtos com } \\
\text { fornecedores, através } \\
\text { de acordos formais }\end{array}$ & & & \\
\hline $\begin{array}{c}\text { Medição e Análise } \\
\text { (MA) }\end{array}$ & & & $\begin{array}{c}\text { Desenvolver e } \\
\text { manter } \\
\text { medições para } \\
\text { apoiar a } \\
\text { gerência }\end{array}$ & \\
\hline $\begin{array}{c}\text { Garantia da } \\
\text { Qualidade de } \\
\text { Processo e } \\
\text { Produto (PPQA) } \\
\end{array}$ & & & $\begin{array}{l}\text { Avaliação de } \\
\text { qualidade do } \\
\text { processo e dos } \\
\text { produtos }\end{array}$ & \\
\hline $\begin{array}{c}\text { Gerência de } \\
\text { Configuração (CM) }\end{array}$ & $\begin{array}{c}\text { Estabelecer e manter } \\
\text { a integridade dos } \\
\text { produtos }\end{array}$ & & & \\
\hline
\end{tabular}

Tabela 3. Mapeamento Práticas da área de processo Gerência de Requisitos.

\begin{tabular}{|c|c|c|c|c|}
\hline \multirow{2}{*}{ CMMI } & Confiabilidade & $\begin{array}{c}\text { Capacidade } \\
\text { de Resposta }\end{array}$ & Segurança & Empatia \\
\cline { 2 - 5 } $\begin{array}{c}\text { PE 1.1 Obter } \\
\text { entendimentos dos } \\
\text { requisitos }\end{array}$ & & $\begin{array}{c}\text { Desenvolver o } \\
\text { entendimento dos } \\
\text { requisitos com os } \\
\text { especificadores }\end{array}$ & \\
\hline $\begin{array}{c}\text { PE 1.2 Obter } \\
\text { comprometimento } \\
\text { com os requisitos }\end{array}$ & $\begin{array}{c}\text { Obter } \\
\text { comprometimento com } \\
\text { os requisitos dos } \\
\text { participantes do projeto }\end{array}$ & \\
\hline $\begin{array}{c}\text { PE 1.3 Gerenciar } \\
\text { as mudanças de } \\
\text { reuisitos }\end{array}$ & $\begin{array}{c}\text { Gerenciar as } \\
\text { mudanças nos } \\
\text { requisitos }\end{array}$ & & & $\begin{array}{c}\text { Gerenciar as } \\
\text { mudanças nos } \\
\text { requisitos }\end{array}$ \\
\hline $\begin{array}{c}\text { PE 1.5 Identificar } \\
\text { inconsistências } \\
\text { entre o trabalho do } \\
\text { projeto e os } \\
\text { requisitos }\end{array}$ & $\begin{array}{c}\text { Identificar } \\
\text { inconsistências } \\
\text { entre o plano de } \\
\text { projeto, os } \\
\text { produtos e os } \\
\text { requisitos }\end{array}$ & & $\begin{array}{c}\text { Identificar } \\
\text { inconsistências } \\
\text { entre o plano de } \\
\text { projeto, os } \\
\text { produtos e os } \\
\text { requisitos }\end{array}$ \\
\hline
\end{tabular}


Finalmente, optou-se por utilizar um relacionamento de cada prática específica, não mais com a descrição da dimensão, e sim com as questões dos modelos de qualidade de serviço (SERVQUAL e IS-SERVQUAL), que mais se assemelhavam ao objetivo da prática. Este formato apresentou melhores resultados de correlação entre o CMMI® e os modelos de qualidade de serviço, fazendo com que todas as práticas estivessem relacionadas à pelo menos uma questão dos modelos de qualidade. Um exemplo do tipo de relacionamento empregado é o da prática específica 1.1 da área de processo Gerência de Requisitos. Esta prática trata de obter o entendimento dos requisitos de software, e assim estaria relacionada à questão do SERVQUAL, que procura verificar se os funcionários do provedor são solidários aos problemas apresentados pelo cliente, e às questões equivalentes do SERVQUAL e IS-SERVQUAL que abordam o entendimento das necessidades específicas dos clientes na dimensão de empatia.

\subsection{Construção do Questionário}

A partir do mapeamento definido anteriormente, entre as práticas do $\mathrm{CMMI}{ }^{\circledR}$ e as questões do modelo de qualidade de serviço, foram elaboradas 48 questões (expectativa somente). A confecção dessas questões contou com duas configurações de montagem. A primeira onde foram utilizadas às questões conforme encontradas na tradução dos modelos de qualidade de serviço, apenas aplicando mudanças de termos como "excelente serviço de informação" por "provedor de serviço com excelência" e "usuários" por "clientes" (Tabela 4). A segunda configuração seria uma adaptação maior das questões originais, com itens ou exemplos de produtos e atividades contidos no CMMI®. Como exemplo pode-se tomar a adaptação da questão do IS-SERQUAL, que estava relacionada a diversas áreas, implicou na inclusão dos exemplos das diversas atividades relacionadas no corpo da questão, como pode ser observado na Tabela 4 segundo item.

Tabela 4. Exemplos de utilização das questões

\begin{tabular}{|c|c|c|}
\hline Questão Original & $\begin{array}{c}\text { Configuração } \\
\text { de utilização }\end{array}$ & Resultado final \\
\hline $\begin{array}{c}\text { I13 - Funcionários em um } \\
\text { excelente serviço de } \\
\text { informação irão entender as } \\
\text { necessidades específicas de } \\
\text { seus usuários? }\end{array}$ & Mesma forma & $\begin{array}{c}\text { Funcionários de um provedor de serviço com } \\
\text { excelência irão entender as necessidades } \\
\text { específicas de seus clientes? }\end{array}$ \\
$\begin{array}{c}\text { I7 - O comportamento dos } \\
\text { funcionários em um excelente } \\
\text { serviço de informação irá } \\
\text { inspirar confiança a seus } \\
\text { usuários? }\end{array}$ & Adaptação & $\begin{array}{c}\text { O comportamento dos funcionários de um } \\
\text { provedor com excelência, nas diversas } \\
\text { atividades do serviço (levantamento e } \\
\text { rastreabilidade de requisitos, monitoração do } \\
\text { projeto, soluções técnicas, verificação e } \\
\text { validação dos produtos, análise de riscos, } \\
\text { auditorias de configuração, etc), irá inspirar } \\
\text { confiança em seus clientes? }\end{array}$ \\
\hline
\end{tabular}

Finalmente, para atender a última premissa adotada neste trabalho (limitação da quantidade questões impostas ao instrumento) foram realizados refinamentos nas questões, de forma a agrupar questões semelhantes ou redundantes e adicionar exemplos relacionados às práticas do $\mathrm{CMMI}{ }^{\circledR}$ nas perguntas. Após este refinamento se obteve um conjunto de 26 questões de expectativa que levam a um total de 52 questões a serem 
avaliadas quando são consideras expectativas e percepções. $\mathrm{O}$ formato de resposta utilizado foi em escala do tipo Likert de 7 pontos, com valores de 1, significando discordância total, até 7 a concordância total. Como informações demográficas foram definidas: nome, e-mail, cargo, ramo de atuação, empresa e o centro avaliado de qual é cliente, de forma a garantir que estava realmente analisando centro avaliado.

\subsection{Validação Semântica}

Com o questionário definido, foi realizado uma validação semântica com 5 voluntários com conhecimentos do modelo CMMI®. Os membros faziam parte de diferentes organizações: 3 oriundos de provedores formalmente avaliados no modelo, 1(um) membro da academia e 1 cliente. A reunião foi conduzida como um "brainstorming", tendo duração aproximada de uma hora e vinte minutos. Os primeiros 25 minutos, aproximadamente, foram reservados para que os membros realizassem a leitura individual e anotação de comentários sobre o instrumento. Em seguida, o item demográfico ou questão era lido em voz alta pelo coordenador da seção e os membros se manifestavam caso tivessem algum comentário ou sugestão a fazer.

Como resultado, foi obtida uma lista de 25 sugestões de melhorias: 5 relacionadas a textos introdutórios e campos de dados demográficos do questionário, 14 "genéricas" relacionadas tanto à questões de expectativa quanto de percepção, 3 relacionadas somente a questões de expectativas e mais 3 de somente às de percepção. Todas as sugestões foram acatadas na revisão, dando origem ao instrumento em sua versão para coleta, apresentado na Tabela 5 para os itens de expectativa. As questões de percepção foram elaboradas com mesmo conteúdo mudando apenas o foco. Por exemplo, a questão número 1 , na visão para análise da percepção, foi formulada da seguinte maneira: "Quando seu provedor de serviço promete fazer algo em certo prazo, ele cumpre". As questões foram apresentadas no instrumento de coleta de forma desagrupada das dimensões. A Tabela 5 apresenta o número do item no instrumento de coleta e as áreas de processo do CMMI® com as quais se relacionam.

Tabela 5. Versão final dos itens do instrumento.

\begin{tabular}{|c|c|c|c|}
\hline & $\begin{array}{l}\text { No. } \\
\text { Item }\end{array}$ & Questões de Expectativa & $\begin{array}{c}\text { Área de } \\
\text { Processo CMMI }^{1}\end{array}$ \\
\hline \multirow{7}{*}{ 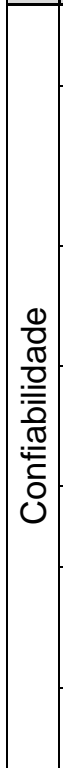 } & 1 & $\begin{array}{l}\text { Quando o provedor de serviço promete fazer algo em certo prazo, } \\
\text { ele irá cumpri-lo. }\end{array}$ & PP,PMC,IPM \\
\hline & 5 & $\begin{array}{l}\text { O provedor de serviço deve ser confiável em relação aos aspectos } \\
\text { técnicos. }\end{array}$ & $\begin{array}{c}\text { SAM,PPQA,RD,T } \\
\text { S,PI,VER,VAL,IP } \\
\text { M,RSKM,OPF,O } \\
\text { T,ISM, DAR } \\
\end{array}$ \\
\hline & 9 & $\begin{array}{l}\text { O provedor de serviço manterá seus produtos/arquivos (plano de } \\
\text { projeto, documentação, código fonte, componentes, registros de } \\
\text { qualidade, etc) organizados. }\end{array}$ & $\begin{array}{l}\text { PP,PMC,MA,TS, } \\
\text { PPQA,CM,PI,RE } \\
\text { QM } \\
\end{array}$ \\
\hline & 13 & $\begin{array}{l}\text { O provedor de serviço será solidário e tranqüilizador nas atividades } \\
\text { de interação com os clientes (entendimento dos requisitos, controle } \\
\text { de mudanças, monitoração dos riscos, etc). }\end{array}$ & PMC,REQM \\
\hline & 17 & $\begin{array}{l}\text { O provedor de serviço irá entreg } \\
\text { foi prometido. }\end{array}$ & PP,PMC,IPM \\
\hline & 21 & $\begin{array}{l}\text { Os funcionários do provedor irão verificar se os requisitos do } \\
\text { serviço estão compatíveis com restrições de custo, tempo, } \\
\text { desempenho, etc. }\end{array}$ & $\mathrm{RD}$ \\
\hline & 25 & $\begin{array}{l}\text { O provedor de serviço irá executar de forma tecnicamente correta } \\
\text { o serviço. }\end{array}$ & TS,PI \\
\hline
\end{tabular}


Tabela 5 (cont.). Versão final dos itens do instrumento.

\begin{tabular}{|c|c|c|c|}
\hline & $\begin{array}{l}\text { No. } \\
\text { Item }\end{array}$ & Questões de Expectativa & $\begin{array}{c}\text { Área de } \\
\text { Processo CMMI }\end{array}$ \\
\hline \multirow{6}{*}{ 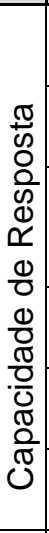 } & 22 & $\begin{array}{l}\text { Funcionários do provedor de serviço irão fornecer um pronto } \\
\text { tratamento dos riscos. }\end{array}$ & PP,RSKM \\
\hline & 18 & $\begin{array}{l}\text { Funcionários do provedor de serviço sempre estarão disponíveis } \\
\text { para responder as requisições dos clientes. }\end{array}$ & $P P, R D$ \\
\hline & 14 & $\begin{array}{l}\text { Os funcionários do provedor de serviço estarão sempre de boa } \\
\text { vontade para identificar as necessidades específicas de seus } \\
\text { clientes. }\end{array}$ & RD,REQM,PMC \\
\hline & 10 & $\begin{array}{l}\text { O provedor de serviço terá controle de todos os planos relativos ao } \\
\text { projeto. }\end{array}$ & IPM \\
\hline & 6 & $\begin{array}{l}\text { É esperado que o provedor de serviço apresente aos clientes uma } \\
\text { estimativa de esforço e custo do serviço. }\end{array}$ & PP \\
\hline & 2 & $\begin{array}{l}\text { E esperado que o provedor de serviço diga aos clientes } \\
\text { exatamente quando os serviços serão concluídos. }\end{array}$ & PMC \\
\hline \multirow{6}{*}{ 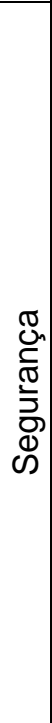 } & 3 & $\begin{array}{l}\text { O comportamento profissional dos funcionários do provedor, nas } \\
\text { diversas atividades do serviço (levantamento e rastreabilidade de } \\
\text { requisitos, monitoração do projeto, soluções técnicas, verificação e } \\
\text { validação dos produtos, etc), irá inspirar confiança em seus } \\
\text { clientes. }\end{array}$ & $\begin{array}{l}\text { REQM,PMC,RD, } \\
\text { TS,VER,VAL,OP } \\
\text { F,OT,PI,IPM,ISM, } \\
\text { DAR, RSKM,CM }\end{array}$ \\
\hline & 7 & $\begin{array}{l}\text { Os funcionários do provedor de serviço terão suporte adequado } \\
\text { (orientações para o planejamento do projeto, documentos e } \\
\text { procedimentos para aquisição, treinamentos, procedimentos para } \\
\text { verificação e validação, etc) para executar bem seus trabalhos. }\end{array}$ & $\begin{array}{c}\text { REQM,PP,SAM, } \\
\text { MA,PPQA,CM,PI, } \\
\text { VER,VAL,OPD,IP } \\
\text { M,DAR } \\
\end{array}$ \\
\hline & 11 & $\begin{array}{l}\text { O provedor de serviço irá executar acompanhamento periódico do } \\
\text { projeto. }\end{array}$ & PMC \\
\hline & 15 & $\begin{array}{l}\text { Os funcionários do provedor de serviço irão inspirar confiança na } \\
\text { identificação de problemas e definição de ações corretivas. }\end{array}$ & PCM \\
\hline & 19 & $\begin{array}{l}\text { Os funcionários do provedor de serviço terão o conhecimento } \\
\text { necessário para responder às questões dos clientes. }\end{array}$ & PP,OT \\
\hline & 23 & $\begin{array}{l}\text { Os clientes irão se sentir seguros em suas negociações de prazo, } \\
\text { custo, escopo, revisões de marcos, etc. com funcionários do } \\
\text { provedor de serviço. }\end{array}$ & $\begin{array}{l}\text { REQM,IPM,PP,P } \\
\text { MC }\end{array}$ \\
\hline \multirow{6}{*}{ 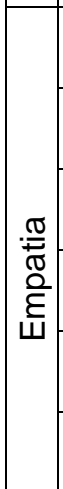 } & 24 & $\begin{array}{l}\text { Funcionários do provedor de serviço irão entender as } \\
\text { necessidades específicas de seus clientes. }\end{array}$ & REQM,PP,RD \\
\hline & 20 & $\begin{array}{l}\text { O provedor de serviço irá fornecer aos seus clientes atenção } \\
\text { individualizada. }\end{array}$ & PP,RD,PMC,IPM \\
\hline & 16 & $\begin{array}{l}\text { Funcionários do provedor de serviço estarão comprometidos com } \\
\text { o plano de projeto para o serviço. }\end{array}$ & PP \\
\hline & 12 & $\begin{array}{l}\text { O provedor de serviço estará preocupado em manter o } \\
\text { comprometimento com os clientes. }\end{array}$ & REQM,PMC \\
\hline & 8 & $\begin{array}{l}\text { Os funcionários do provedor de serviço serão capazes de traduzir } \\
\text { o levantamento de necessidades em requisitos de software. }\end{array}$ & $\mathrm{RD}$ \\
\hline & 4 & $\begin{array}{l}\text { O provedor de serviço irá solicitar a participação do cliente nas } \\
\text { atividades relevantes do projeto. }\end{array}$ & IPM \\
\hline
\end{tabular}

'Legenda areas do nível 3 (nível 2 na Tabela 2): RD - Requirements Development, TS Technical Solution, PI - Product Integration, VER - Verification, VAL - Validation, OPF Organization Process Focus, OPD - Organization Process Definition, OT - Organizational Training, IPM - Integrated Project Management, RM - Risk Management, ISM - Integrated Supplier Management, DAR - Decision Analysis and Resolution. 


\section{Aplicação do Instrumento}

A aplicação do instrumento ocorreu no Distrito Federal, onde o público alvo, clientes ou potenciais clientes de empresas formalmente avaliadas nos modelos CMM®/CMMI®, estavam sediados. Não houve restrição alguma quanto à localização do provedor de serviço.

O instrumento construído foi disponibilizado para preenchimento dos respondentes de forma eletrônica, em um sítio na Internet (www.qualidadedeservicocmmi.eti.br). Tais respondentes foram selecionados primariamente pelo seu perfil (cliente ou possíveis clientes), do círculo de relacionamento dos autores. Todos os respondentes foram contatados por e-mail, no qual foi apresentado os objetivos da pesquisa, os perfis necessários para a participação e informações de localização do sitio para preenchimento.

Existiam dois questionários disponíveis, um para respondentes que nunca foram clientes de empresas formalmente avaliadas no $\mathrm{CMM} \circledast / \mathrm{CMMI} \AA$, contudo possuíam conhecimentos acerca de tais modelos, e um segundo questionário para os respondentes que já foram clientes de empresas formalmente avaliadas nos modelos. O objetivo do questionário de não clientes era unicamente identificar as expectativas de possíveis clientes acerca das empresas formalmente avaliadas e, portanto, contou apenas com os itens de expectativa do instrumento proposto. Já o questionário de clientes tinha por objetivo avaliar a qualidade percebida do serviço (Percepção - Expectativa) e contou com a forma completa do instrumento ( 25 itens para avaliação das expectativas e 25 para percepção).

\subsection{Analise dos Resultados}

Foram obtidas 78 (setenta e oito) respostas para o questionário de não clientes e 42 (quarenta e duas) para o questionário de clientes das empresas formalmente avaliadas nos modelos $\mathrm{CMM} / \mathrm{CMMI}$.

Para o questionário de não clientes, a distribuição demográfica dos participantes em relação ao cargo, indica um equilíbrio entre os cargos analistas (23\%), gerente de área $(21 \%)$, consultor (21\%) e líder de projeto (20\%). Os cargos desenvolvedor e gestor de contrato obtiveram um menor representação com $3 \%$ cada um. No ramo de atuação, a maioria se posicionou nas áreas bancárias/financeiras $(55 \%)$ e de tecnologia da informação $(21 \%)$, porém com representação também nas áreas de administração pública (10\%), telecomunicações (10\%) e educacional (4\%).

Já no questionário de clientes $\mathrm{CMM} 囚 / \mathrm{CMMI} \AA$, os cargos de maior prevalência foram analistas $(25 \%)$, gerente de área $(21 \%)$, consultores $(21 \%)$, líderes de projeto $(17 \%)$ e outros $(12 \%)$. Houve também respostas de desenvolvedores e gestores de contrato com representação de $2 \%$ cada Para ramo de atuação, também prevaleceu as áreas bancárias/financeiras (54\%) e de tecnologia da informação (27\%) com os maiores valores, sendo o restante $(15 \%)$ da área de telecomunicações, varejo (2\%) e administração pública (2\%).

Aplicando o teste de comparação de média (Teste t de Student), o primeiro dado importante identificado nesta amostra, foi que não houve diferença significativa $(\mathrm{t}=$ 0,833) com nível de significância $(\mathrm{p})>0,05$, entre os itens de expectativa dos dois 
questionários. Esta análise demonstra que as expectativas de clientes e não clientes estão em patamares semelhantes não sendo afetada pela prestação do serviço. Esta constatação permitiu que os itens de expectativa dos dois questionários fossem agrupados para uma análise conjunta $(n=120)$.

$\mathrm{Na}$ análise conjunta dos dados de expectativa, utilizando-se o procedimento de analise de variância (ANOVA) com teste de hipótese nula $\mathrm{F}$, não foram identificadas diferenças significativas entre as expectativas e os cargos $(F=1,092$ com $p>0,05)$, e expectativas e ramo de atuação $(F=1,730 \mathrm{com} p>0,05)$.

Ponderando sobre as médias de cada item de expectativa, pôde-se observar que os itens $4,11,9,12,2,3$, e 5 foram os avaliados com maior qualidade esperada pelos respondentes ( $\mu$ expectativa $>5,5$ ). Segundo o mapeamento executado na atividade de construção do instrumento, é possível identificar que: (i) as questões 2, 4 e 11, mantém relação com atividades relacionadas a gerência integrada (IPM) e controle e monitoração de projetos (PMC); (ii) a questão de número 12, indicaria uma preocupação com o comprometimento do provedor com seus clientes e estaria relacionada às área de gerência de requisitos e também PMC; e, (iii) as questões 9, 3 e 5, tratam de aspectos técnicos que um provedor deveria atender durante a prestação de um serviço como: monitoração de projeto, verificação, validação, controle da rastreabilidade, gerência de configuração, etc.

Analisando a situação oposta, ou seja, itens que tiveram os valores médios de avaliação com os menores números, não foi observado nenhum item onde o valor médio estivesse abaixo da mediana (4) dos valores disponíveis na escala de respostas ( 1 a 7 ). $\mathrm{O}$ item que mais se aproximou $(\mu=4,39)$ da mediana foi a questão 20 (vinte), que trata do recebimento pelo cliente de atenção individualizada do provedor de serviço, que se relaciona com quatro áreas do modelo: PP, RD, PMC e IPM, com maior ênfase no planejamento de projeto.

$\mathrm{Na}$ análise das respostas com as percepções dos clientes $(\mathrm{n}=42)$, novamente utilizando ANOVA com teste de hipótese nula $\mathrm{F}$, não foram identificadas diferenças significativas entre as percepções e os cargos $(F=2,72$, com $p>0,05)$. Já para o relacionamento com os ramos de atuação, utilizando a técnica de comparações múltiplas de Scheffé que busca maior proteção em relação a falsos alarmes, foi observado uma diferença significativa entre os cargos "Bancária/Financeira" e "TI - Tecnologia da Informação" (1,29 pontos médios, com $\mathrm{p}<0,05)$. Vale destacar que não houve respondente com os ramos " Educacional" e "Indústria" e que os ramos "Administração Pública", "Varejo" e "Outros", por possuírem menos de dois eventos, foram retirados da análise. Contudo este dado pode ter siso influenciado pelo fato de que 72,72\% (8) dos respondentes do ramo de atuação "Bancária/Financeira" terem avaliado a mesma empresa e centro CMMI®.

Para o campo demográfico tempo de prestação de serviço $(F=0,743$ com $p$ > 0,05), também não foram encontradas diferenças significativas nas percepções.

$\mathrm{Na}$ análise da percepção da qualidade de serviço (Percepção(P)-Expectativa(E)), prevista no modelo SERVQUAL, observou-se que de forma geral a qualidade de serviço percebida pelos clientes não é satisfatória (Figura 1 e 2), com apenas a questão $20 \mathrm{com}$ valores $\geq 0$, que seriam os adequados. Esta questão lida com a atenção individualizada, 
e estaria relacionada a algumas práticas das áreas PP, RD, PMC e IPM. Todavia os itens 14, 18 e 19 chegaram próximos (P-E > - 0,5) de alcançar os valores esperados pelos clientes. A questão $14(\mathrm{P}-\mathrm{E}=-0,41)$ trata da boa vontade na identificação das necessidades específicas dos clientes, e está relacionada as áreas de gerenciamento de requisitos (REQM), RD e PMC. A questão 18 , que possui a menor diferença (P-E = 0,09), está relacionada a área de $\mathrm{PP}$ e $\mathrm{RD}$, particularmente para disponibilidade de atendimento das requisições do cliente. Já a questão 19 (P-E = -0,47), trata do conhecimento necessário dos funcionários do provedor para desempenhar as tarefas previstas no serviço, e se relaciona com as áreas PP e treinamento organizacional (OT).

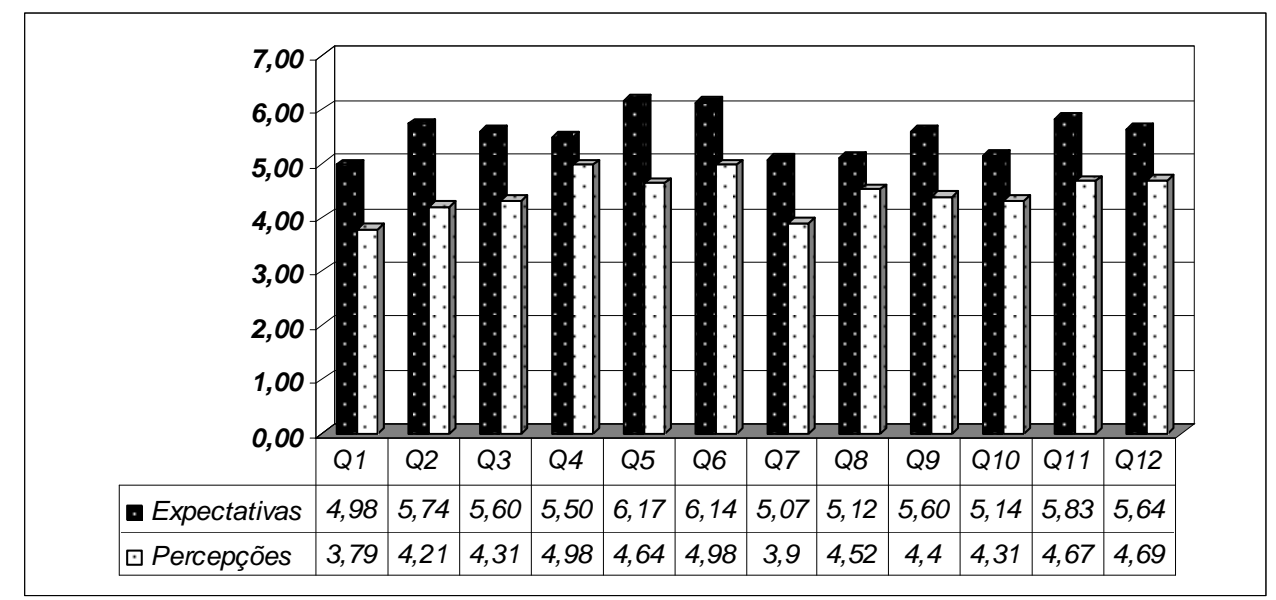

Figura 1. Comparativo entre expectativa e percepção (Itens de 1 a 12).

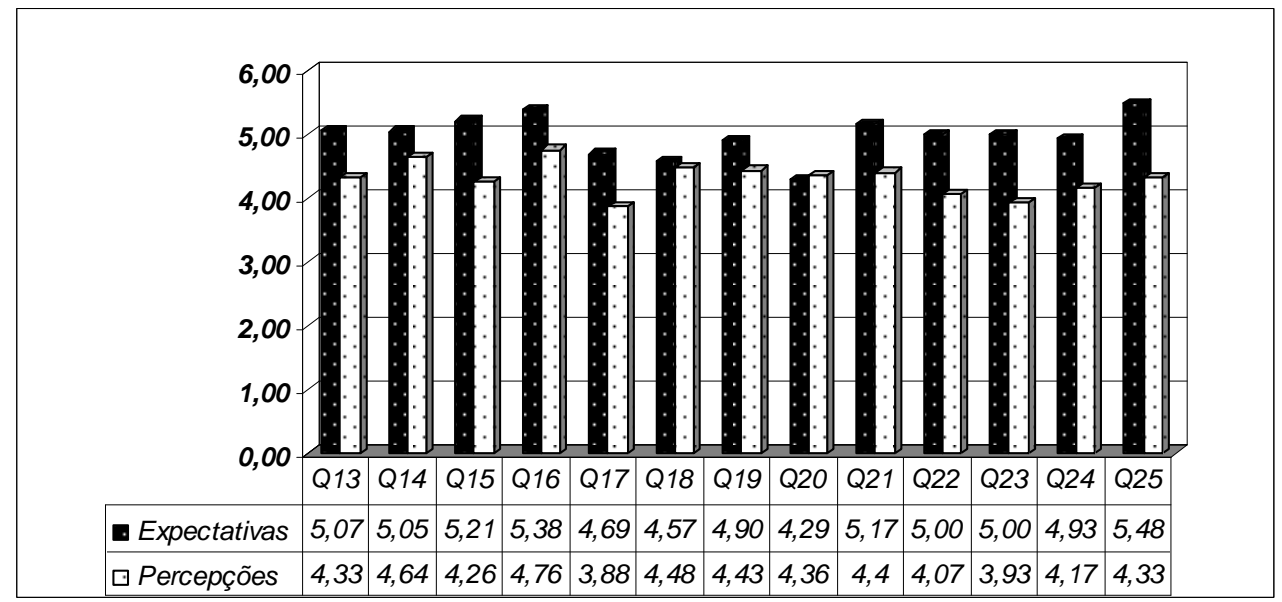

Figura 2. Comparativo entre expectativa e percepção (Itens de 13 a 25).

Os itens 2 , e 5 se destacaram com valores de percepção bem mais baixos que os de expectativa, $(\mathrm{P}-\mathrm{E}<-1,5)$, apresentando mesmo resultado de $\mathrm{P}-\mathrm{E}=-1,53$. O item 2 avalia o repasse de informações acerca do prazo de conclusão dos serviços, relacionado a área PMC. Já o item 5 avalia a confiança na capacidade técnica do provedor, e estaria relacionada a diversas áreas como: gerência de aquisição de software, verificação, validação, desenvolvimento de requisitos, entre outras.

No geral o instrumento se apresentou confiável exibindo, por exemplo, na análise de intercorrelação das dimensões do questionário de clientes por Alfa de Cronbach, valores acima de 0,9 (Confiabilidade $=0,95$, Capacidade de Resposta = 0,90, 
Segurança $=0,93$, Empatia $=0,89$ ), o quê segundo Pasquali (2002) são valores que demonstram a confiabilidade dos fatores.

\section{Conclusão}

Neste artigo foi apresentado um instrumento de avaliação da qualidade de serviço, para provedores formalmente avaliados nos modelos $\mathrm{CMM} \circledast / \mathrm{CMMI} \AA$, baseado no SERVQUAL e uma de suas derivações o IS-SERVQUAL. Este instrumento se apresentou confiável em relação as suas dimensões e itens.

Com a massa de dados disponível, este estudo identificou alguns resultados importantes, entre eles:

- Clientes e possíveis clientes possuem avaliações semelhantes em relação à expectativa, o quê sugere que o nível de exigência não se eleva quando um indivíduo se torna cliente;

- Clientes e possíveis clientes possuem alta expectativa em relação aos provedores de serviço, principalmente nas atividades de gerenciamento, controle e planejamento de projeto e em atividades de aspectos técnicos como verificação, validação, gerência de configuração, etc.

- A percepção dos clientes em relação aos serviços prestados demonstra um desalinhamento em relação às expectativas, principalmente sobre o repasse de informações acerca do prazo de conclusão dos serviços.

Como trabalhos futuros, estão previstos a realização de análises com um maior número de respondentes e outros cruzamentos de informações, como por exemplo, se o nível de maturidade, ou a versão do modelo utilizada (CMM®/CMMI®) do provedor de serviço possui alguma correlação com a expectativa e/ou percepção dos clientes.

\section{Agradecimentos}

Os autores agradecem M.Sc. Maja Meira pelo apoio fundamental para avaliação estatística dos resultados e a todos os respondentes da avaliação, sem os quais não seria possível a concretização desse trabalho.

\section{Referências}

Ahren, D. M., Clouse, A. e Turner R (2003). "CMMI® Distilled: A Practical Introduction to Integrated Process Improvement” Boston, Addison Wesley, $2^{\mathrm{a}}$ edição, 336 páginas.

Associação Brasileira de Normas Técnicas (2003). "NBR ISO/IEC 9126-1: Engenharia de Software - Qualidade do Produto" Rio de Janeiro, ABNT, 21 páginas.

Bevan, N. (1999). "Quality in use: Meeting user needs for quality" The Journal of Systems and Software, vol. 48, n. 1, páginas 89-96.

Brasil, Ministério da Ciência e Tecnologia (2006). "Qualificação CMM e CMMI no Brasil”, Brasília, http://www.mct.gov.br/upd_blob/9238.pdf, Acesso em: 15 fev. 2007. 
Carnegie Mellon University, Software Engineering Institute (2002a). "Capability Maturity Model ${ }^{\circledR}$ Integration (CMMISM),Version 1.1. CMMISM for Systems Engineering, Software Engineering, Integrated Product and Process Development, and Supplier Sourcing (CMMI-SE/SW/IPPD/SS, V1.1), Staged Representation" Pittsburgh, 711 páginas.

Software Engineering Institute (2002b). "Capability Maturity Model $®$ Integration (CMMISM),Version 1.1. CMMISM for Systems Engineering, Software Engineering, Integrated Product and Process Development and Supplier Sourcing (CMMI-SE/SW/IPPD/SS, V1.1), Continuous Representation” Pittsburgh, 711 páginas.

, Software Engineering Institute (2007). "Published CMMI® Appraisal Results" Pittsburgh, http://sas.sei.cmu.edu/pars/pars.aspx, Acesso em: 15 fev. 2007.

CMMI, Capability Maturity Model® Integration (2006). "Apresenta resultados de desempenho coletados a partir de avaliações do modelo CMMI até 15 de Dezembro de 2005" Desenvolvido por Carnegie Mellon University, Software Engineering Institute, http://www.sei.cmu.edu/CMMI/results.html, Acesso em: 04 abr. 2006.

Chrissis, M, B., Konrad, M. e Shrum, S. (2003). "CMMI®: Guidelines for Process Integration and Product Improvement” Boston, Addison Wesley, 688 páginas.

Cronin, J. J. Jr. e Taylor, S. A. (1992). "Measuring service quality: a reexamination and extension” Journal of Marketing, vol. 56, n. 3, páginas. 55-68.

Franceschini, R. (2001). "Qualidade dos serviços públicos: como dimensionar?" Agência Estadual de Regulação dos Serviços Públicos Delegados do Rio Grande do Sul, http://www.agergs.rs.gov.br/bibliot/revista/mr3/mr3_renzo.htm, Acessado em: 27 de maio de 2006.

Grönroos, C. (1993). "Marketing: Gerenciamento e Serviços: A competição por serviços na hora da verdade" Rio de Janeiro, Campus, 13ª edição, 337 páginas.

Integrated System Diagnostic Brasil (2007). "Maturidade do Mercado Nacional" São Paulo, http://www.isdbrasil.com.br/NossosCli/maturicli.htm, Acesso em: 15 fev. 2007.

Kang, H. e Bradley, G. (2002) "Measuring the performance of IT services: An assessment of SERVQUAL" International Journal of Accounting Information Systems, vol. 3, n. 3, páginas 151-164.

Kettinger, W. J. e Lee, C. C. (1997) "Pragmatic Perspectives on the Measurement of Information Systems Service Quality” MIS Quarterly, vol. 21, n. 2, páginas 223-240.

Kulpa, M. K. e Johson, K. A. (2003). "Interpreting the CMMI: A Process Improvement Approach” Boca Raton, Auerbach Publications, 414 páginas.

Paiva, C. C. S. de (2001). "Nível de qualidade dos serviços bancários no ambiente digital" Dissertação (Mestrado em Engenharia de Produção) - Universidade Federal de Santa Catarina, Florianopolis, 115 páginas. 
Parasuraman, A., Zeithaml, V. A. e Berry, L. L. (1985). "A Conceptual Model of Service Quality and Its Implications for Future Research" Journal of Marketing, vol. 49, n.4, páginas 41-50.

(1988). "SERVQUAL: A Multiple-Item Scale for Measuring Consumer Perceptions of Service Quality" Journal of Retailing, vol. 64, n. 1, páginas 12-40.

Parasuraman, A., Berry, L. L. e Zeithaml, V. A. (1991a). "Understanding Customer Expectations of Service” Sloan Management Review, vol. 32, n. 3, páginas 39-48

(1991b). "Refinement and reassessment of the SERVQUAL scale" Journal of Retailing, vol. 67, n.4, páginas 420-431.

Parasuraman, A., Zeithaml, V. A. e Berry, L. L. (1994). "Reassessment of expectations as a comparison standard in measuring service quality: Implications for further research" Journal of Marketing, vol. 58, n.1, páginas 111-124.

Pasquali, Luiz P. (2002). “Análise fatorial para pesquisadores” Brasília, Editora Universidade de Brasília.

Paulk, M. C. et al. (1993). "Capability Maturity ModelSM for Software. Versão 1.1" Pittsburgh, Software Engineering Institute - Carnegie Mellon University, 65 páginas.

Pitt, L. F., Watson, R. T. e Kavan, C. B. (1997). "Measuring Information Systems Service Quality: Concerns for a Complete Canvas" MIS Quarterly, vol. 21, n. 2, páginas 209-221.

Quintela et al. (2006). “Avaliação dos Serviços de Testes de Software em Instituições financeiras com Adoção das Práticas de Teste do Modelo CMMI" Universidade Federal Fluminense, Relatórios de Pesquisa em Engenharia de Produção, Rio de Janeiro, vol. 6, n. 11, páginas 1-26.

Rados, G. J. V. (2005). "Material de Apoio do curso de Planejamento dos Serviços Bibliotecários" Universidade Federal de Santa Catarina, Centro de Ciências da Educação, Departamento de ciências da Informação, Laboratório de Gestão Tecnologia e Informação. Aula 4, http://www.lgti.ufsc.br/planejamento/, Acessado em: 27 de maio de 2006.

Reis, I. C. da S. (2001). "A escala SERVQUAL modificada: Avaliação da qualidade percebida do serviço de lazer oferecido por um complexo poliesportivo num parque florestal" Florianopolis, Dissertação (Mestrado em Engenharia de Produção) Universidade Federal de Santa Catarina, 141 páginas.

Slack, N. et al. (1996). “Administração da Produção” São Paulo, Atlas, $1^{\circ}$ edição, 726 páginas.

Softex (2006). "MPS.BR - Melhoria de Processo do Software Brasileiro”, 56 páginas.

Van Dyke, T. P., Kappelman, L. A. e Prybutok, V. R. (1997). "Measuring Information Systems Service Quality: Concerns on the Use of the SERVQUAL Questionnaire" MIS Quarterly, vol. 21, n. 2, páginas 195-208. 